\title{
Detección del riesgo para diabetes tipo 2 y factores asociados en trabajadores de Villavicencio
}

\section{Detecting the risk for type 2 diabetes and associated factors in workers of Villavicencio}

\author{
Harold Suárez-Alfaro ${ }^{1 *}$ y Norton Pérez-Gutiérrez ${ }^{2}$
}

${ }^{1}$ Facultad de Ciencias de la Salud, Universidad de los Llanos; ${ }^{2}$ Facultad de Medicina, Universidad Cooperativa de Colombia. Villavicencio, Meta, Colombia

\section{RESUMEN}

Introducción: La prevención de la diabetes requiere la identificación de individuos con riesgo aumentado para desarrollarla. El cuestionario FINDRISC (FINnish Diabetes RIsk SCore) permite identificarlos. Objetivo: Identificar el riesgo para desarrollar diabetes mellitus tipo 2 y sus factores asociados en una población de trabajadores. Metodología: Estudio analítico de corte transversal. Se incluyeron 449 trabajadores adultos, de ambos sexos. Se aplicó el cuestionario FINDRISC, preguntas sobre consumo de bebidas azucaradas y tiempo en posición sedente. Resultados: El 19.8\% de los trabajadores tienen alto riesgo para diabetes. Entre los sujetos con alto riesgo para diabetes el consumo promedio de bebidas azucaradas fue 3.25 veces/día, el promedio de tiempo diario en posición sedente fue de 3.8 horas/día, la media del índice de masa corporal fue 31.1. Se encontró una asociación estadísticamente significativa entre el consumo de diario de bebidas azucaradas y el riesgo para desarrollar diabetes (OR: 5.07; $\mathrm{p}<0.05)$. Conclusiones: Una proporción elevada de trabajadores tienen alto riesgo para diabetes, este riesgo estuvo asociado con el consumo de bebidas azucaradas.

Palabras clave: Diabetes. Factores de riesgo. Salud ocupacional. Prevención. Bebidas azucaradas.

\section{ABSTRACT}

Introduction: Prevention of diabetes requires identification of individuals at increased risk for developing it. The FINDRISC questionnaire allows to identify them. Objective: To identify the risk of developing type 2 diabetes mellitus and its associated factors in workers. Methodology: Cross-sectional analytical study. 449 adult workers of both sexes were included. The FINDRISC questionnaire was applied, as well as questions on consumption of sweetened beverages and time in a sedentary position. Results: $19.8 \%$ of workers are at high risk for diabetes. Among the subjects with high risk, average consumption of sweetened beverages was 3.25 times/day, the average time in sedentary position was 3.8 hours/day, and the average body mass index was 31.1. A statistically significant association was found between daily consumption of sweetened beverages and the risk of developing diabetes (OR: 5.07; $\mathrm{p}<0.05$ ). Conclusions: $A$ high proportion of workers are at high risk for diabetes; this risk is associated with daily consumption of sweetened beverages.

Key words: Diabetes. Risk factors. Occupational health. Prevention. Sweetened beverages. DOI: 10.24875/ALAD.20000022
Disponible en internet: 04-11-2020 Rev ALAD. 2021;11:18-28

2248-6518 / @ 2020 Asociación Latinoamericana de Diabetes. Publicado por Permanyer. Este es un artículo open access bajo la licencia CC BY-NC-ND (http://creativecommons.org/licenses/by-nc-nd/4.0/). 


\section{INTRODUCCIÓN}

Para el año 2019 se estimaban 463 millones de adultos con diabetes mellitus en el mundo1. Por las características propias de la patología y por el requerimiento de pruebas de laboratorio para el diagnóstico, cerca del 50\% de los pacientes diabéticos desconocen su condición ${ }^{2}$. Como consecuencia del diagnóstico tardío y las dificultades en el control de los pacientes, la diabetes continua ocasionando gran cantidad de complicaciones, discapacidad y una elevada mortalidad ${ }^{3}$. Las proyecciones para la próxima década estiman que para el año 2030 habrá más de 578 millones de personas con diabetes en el mundo ${ }^{1}$. Este incremento ocurrirá, principalmente, a expensas de población joven en los países con medianos y bajos ingresos. En Colombia para el año 2019 se estimaban más de 2.8 millones de adultos afectados ${ }^{1}$. Las proyecciones nacionales indican un rápido incremento en el número de diabéticos con afectación de sujetos cada vez más jóvenes ${ }^{4-5}$.

La diabetes mellitus tipo 2 ( DM 2) genera un gran impacto sanitario, económico y social a nivel global. En consecuencia, la prevención de esta patología se ha convertido en una prioridad para la Organización Mundial de la Salud ${ }^{3}$. Gran número de países han adoptado estrategias para tamización y prevención primaria de la diabetes ${ }^{6}$. Los biomarcadores son indicadores tardíos de la progresión de esta enferme$\mathrm{dad}^{4}$, por tanto, las estrategias basadas en pruebas de laboratorio retardan la implementación de actividades preventivas7. Como alternativa de acción se ha propuesto la identificación de los sujetos en riesgo para desarrollar diabetes sin necesidad de pruebas de laboratorio ${ }^{8,9}$. Dicha estrategia se basa en el reconocimiento de los factores de riesgo para comenzar precozmente con intervenciones que permitan controlarlos ${ }^{10}$. Las herramientas recomendadas para la identificación de los sujetos en riesgo para diabetes son aquellas basadas en cuestionarios $^{11}$. En Colombia, la Guía de práctica clínica para la diabetes mellitus ${ }^{12}$ y el Consenso de expertos sobre prediabetes ${ }^{11}$ recomiendan utilizar el cuestionario FINDRISC (FINnish Diabetes RIsk SCore) para identificar adultos en riesgo para diabetes en todos los niveles del sistema de salud ${ }^{8}$. El cuestionario FINDRISC es una herramienta práctica, fácil de aplicar y altamente costo-efectiva para identificar sujetos en riesgo para diabetes ${ }^{8,10,13}$.

Con el propósito de garantizar la salud de los trabajadores, en Colombia se realizan de forma continua y sistemática exámenes médicos ocupacionales a los trabajadores al momento de la vinculación laboral y periódicamente durante la vigencia del contrato laboral14,15. Los exámenes para trabajadores, que se realizan en centros médicos especializados en salud ocupacional, brindan una oportunidad óptima para la aplicación de instrumentos que permitan identificar factores de riesgo y tamizar a grandes grupos poblacionales ${ }^{6}$.

La ciudad de Villavicencio, ubicada en la zona suroriental colombiana y con aproximadamente 451,000 habitantes, aporta gran parte de la fuerza laboral requerida por la industria de la región. El objetivo principal de este estudio es identificar el riesgo para desarrollar DM2 y sus factores asociados en una población de trabajadores en Villavicencio durante el último bimestre de 2019.

\section{MATERIALES Y MÉTODOS}

Se realizó un estudio epidemiológico de tipo observacional analítico, de corte transversal y de fuente primaria. La muestra se obtuvo a partir de trabajadores que fueron examinados en un centro médico de salud ocupacional en la ciudad de Villavicencio, durante el último bimestre de 2019. Se incluyeron 
sujetos adultos, de ambos sexos y sin antecedente personal de diabetes. Se excluyeron las pacientes embarazadas. El centro médico de salud ocupacional seleccionado para este estudio es una clínica de carácter privado, de primer nivel de complejidad, con sede principal en Villavicencio y que atiende a trabajadores provenientes de empresas de diferentes sectores productivos.

El marco muestral estuvo conformado por 2,200 trabajadores que son atendidos cada bimestre en el centro médico ocupacional. Se calculó el tamaño muestral tomando como valor de referencia una proporción esperada del $14.4 \%$ de individuos con alto riesgo para desarrollar diabetes ${ }^{16}$. Se trabajó con un nivel de confianza del $95 \%$ y un margen de error aceptable del 3\%. Con el propósito de verificar el proceso de muestreo, la metodología, la precisión y reproductibilidad en la recolección de datos se realizó una prueba piloto que incluyó a 15 trabajadores seleccionados al azar. Los individuos de la prueba piloto no fueron incluidos en el análisis final. Se realizó un muestreo aleatorio sistemático. A los trabajadores que cumplían con los criterios de inclusión se les explicaron los objetivos de la investigación y el manejo confidencial que se daría a la información. Todos los trabajadores incluidos en la muestra manifestaron su aceptación a participar en el estudio y firmaron otorgando su consentimiento informado. La muestra final incluida en el análisis estuvo integrada por 449 trabajadores.

A cada individuo incluido en la muestra se le realizó un examen médico ocupacional completo. Durante la anamnesis se aplicó el cuestionario FINDRISC validado para Colombia ${ }^{12}$. Este cuestionario está compuesto por seis preguntas y dos mediciones corporales, cada variable asigna una puntuación independiente, la sumatoria de los puntajes parciales se presenta como un puntaje total entre o y 26. El resultado final permite clasificar en categorías de riesgo para desarrollar diabetes en los próximos 10 años. La interpretación recomendada para la versión validada en Colombia establece el punto de corte en 12: los individuos que obtengan un puntaje menor a 12 se clasifican con bajo riesgo y aquellos con puntaje igual o mayor a 12 se clasifican en la categoría de alto riesgo. Adicionalmente se aplicó una pregunta sobre frecuencia de consumo de bebidas azucaradas tomada de la Encuesta Nacional de Situación Nutricional 2015. Las bebidas consideradas fueron: gaseosas, bebidas energizantes, jugos procesados industrialmente, jugos naturales y «agua de panela». Se consideró consumo excesivo de bebidas azucaradas cuando un trabajador declaraba que consumía cinco o más bebidas azucaradas por día. Finalmente, se aplicó una pregunta sobre el tiempo diario en posición sedente, tomada del cuestionario mundial sobre actividad física. Se consideró alto nivel de sedentarismo cuando un trabajador pasaba cuatro o más horas al día en posición sedente. Durante el examen físico se tomó el peso corporal usando una báscula calibrada, con el paciente de pie, sin zapatos y con la menor cantidad de prendas de vestir. Se midió la estatura con una regla de pared, con el paciente de pie, sin zapatos, con espalda recta y mirando al frente. Se calculó el índice de masa corporal (IMC). Se clasificó el estado nutricional según los puntos de corte de la Organización Mundial de la Salud. La medición del perímetro abdominal (PA) fue realizada con el paciente de pie, con el abdomen relajado en espiración, pasando la cinta métrica de manera horizontal por el punto medio entre el reborde inferior de la última costilla y el reborde superior de la cresta iliaca. La obesidad abdominal fue definida como el PA aumentado por encima de los puntos de corte sugeridos por la Guía colombiana de sobrepeso y obesidad (para hombres $\geq 94 \mathrm{~cm}$ y para mujeres $\geq 90 \mathrm{~cm})^{17}$. La información se registró en el instrumento para recolección de información impreso en papel. 
Se construyó una base de datos con los registros completos de 449 individuos. Se incluyeron datos demográficos como sexo, estado civil, lugar de residencia, estrato socioeconómico, ultimo nivel educativo terminado y tipo de cargo laboral. Se incluyeron las respuestas al cuestionario FINDRISC: edad, práctica habitual de actividad física, consumo habitual de frutas y verduras, consumo de medicamentos antihipertensivos, antecedente familiar de diabetes, antecedente personal de hiperglucemia y las mediciones antropométricas (peso, talla, IMC y PA). Se incluyeron las respuestas sobre frecuencia de consumo de bebidas azucaradas y número de horas por día en posición sedente. Las variables cuantitativas se presentan como promedio con su desviación estándar y fueron comparadas con la prueba $t$ de Student con una significancia de $p<0.05$. Las variables categóricas se presentan como frecuencias absolutas y frecuencias relativas, se sometieron a prueba de independencia con respecto a la variable resultado mediante el estadístico Chi cuadrada $\left(\chi^{2}\right)$ con una significancia de $p<0.05$. La variable de resultado, riesgo para desarrollar $\mathrm{DM} 2$, se estableció como categórica dicotómica: alto o bajo riesgo. Esta clasificación se realizó según los puntajes obtenidos por cada trabajador en el cuestionario FINDRISC, asumiendo el punto de corte recomendado por la guía colombiana (bajo riesgo: < 12; alto riesgo: $\geq 12$ ). Se informó como frecuencia absoluta y frecuencia relativa con su intervalo de confianza. Las variables independientes fueron categóricas dicotómicas: sexo (hombre/mujer), lugar de residencia (urbano/rural), tipo de cargo (operativo/administrativo), consumo excesivo de bebidas azucaradas (< $5 / \geq 5$ ) y nivel de sedentarismo $(<4 / \geq 4 \mathrm{~h}$ ). Se realizaron los análisis estadísticos pertinentes. La asociación entre el riesgo para diabetes y las variables independientes se investigó mediante análisis bivariante y multivariante con regresión logística, se reportó como odds ratio (OR) ajustada con su intervalo de confianza del 95\% (IC 95\%).
El estudio fue clasificado como una investigación con riesgo mínimo. Se realizó con estricto cumplimiento de las consideraciones éticas para la investigación en seres humanos contenidas en la Declaración de Helsinki y en la resolución 8,430 de $1993^{18}$. El protocolo de investigación y el consentimiento informado fueron avalados por el comité de ética de la Universidad de los Llanos. Se garantizó la confidencialidad en el manejo de la información. A cada trabajador se le entregó copia de su resultado final en el cuestionario FINDRISC y se le realizó una breve explicación con un instructivo para la comprensión de este resultado. A los trabajadores que tuvieron un puntaje igual o mayor de 12 puntos, clasificados con alto riesgo, se les impartió una breve consejería sobre el control de los factores de riesgo identificados y se les recomendó acudir a su servicio médico para realizar las pruebas de laboratorio pertinentes según las recomendaciones nacionales ${ }^{12}$.

\section{RESULTADOS}

La muestra estuvo conformada por 449 individuos adultos.

\section{Análisis univariante}

El rango de edad fue desde 20 hasta 72 años (media: $36 \pm 11$ ). El 72\% (IC 95\%: 68.3-75.7) fueron trabajadores del sexo masculino, el 83\% (IC 95\%: 79.9-86.1) habitantes del área urbana de Villavicencio, el $64 \%$ (IC 95\%: 60.0-67.9) pertenecientes al estrato socioeconómico 2. El 80\% (IC 95\%: 76.7-83.3) se desempeñan en cargos operativos, el 64\% (IC 95\%: 60.0-67.9) tuvieron exceso de peso corporal (42\% sobrepeso $22 \%$ obesidad), el 43\% (IC 95\%: 38.9-47.0) presentan obesidad abdominal (hombres: PA $\geq 94 \mathrm{~cm}$; mujeres: $\mathrm{PA} \geq 90 \mathrm{~cm})$. El promedio global de consumo diario de bebidas azucaradas fue de $2.6( \pm 1.8)$ veces 


\section{Rev ALAD. 2021;11}

por día y el promedio global de tiempo diario en posición sedente fue de $3.3( \pm 2.7)$ horas por día. Las características generales de la muestra evaluada se presentan en la tabla 1.
El 19.8\% (IC 95\%: 16.5-23.1) de los trabajadores incluidos en este estudio fueron clasificados con alto riesgo para desarrollar diabetes. Entre los trabajadores con alto riesgo para diabetes el $53 \%$ supera la edad

TABLA 1. Características generales de la muestra incluida en el estudio ( $\mathrm{n}=449$ )

\begin{tabular}{|c|c|c|c|}
\hline \multicolumn{2}{|l|}{ Variable } & \multirow{2}{*}{$\begin{array}{c}\mathbf{n} \\
341\end{array}$} & \multirow{2}{*}{$\begin{array}{l}\% \\
76\end{array}$} \\
\hline Edad & $<45$ años & & \\
\hline & de 45 a 54 años & 77 & 17 \\
\hline & de 55 a 64 años & 27 & 6 \\
\hline & $\geq 65$ años & 4 & 1 \\
\hline \multirow[t]{2}{*}{ Sexo } & Mujeres & 125 & 28 \\
\hline & Hombres & 324 & 72 \\
\hline \multirow[t]{4}{*}{ Estado civil } & Soltero & 176 & 39 \\
\hline & Unión libre & 163 & 36 \\
\hline & Casado & 104 & 23 \\
\hline & Separado & 6 & 2 \\
\hline \multirow[t]{2}{*}{ Lugar de residencia } & Urbano & 372 & 83 \\
\hline & Rural & 77 & 17 \\
\hline \multirow[t]{4}{*}{ Estrato socioeconómico } & Estrato 1 & 61 & 13 \\
\hline & Estrato 2 & 286 & 64 \\
\hline & Estrato 3 & 96 & 21 \\
\hline & Estrato 4 & 6 & 2 \\
\hline \multirow[t]{4}{*}{ Niel educativo } & Primaria & 37 & 8 \\
\hline & Secundaria & 190 & 43 \\
\hline & Técnico/tecnológico & 131 & 29 \\
\hline & Universitario & 91 & 20 \\
\hline \multirow[t]{2}{*}{ Tipo de cargo laboral } & Administrativo & 90 & 20 \\
\hline & Operativo & 359 & 80 \\
\hline \multirow[t]{2}{*}{ Práctica habitual de actividad física } & Sí & 63 & 14 \\
\hline & No & 386 & 86 \\
\hline \multirow[t]{2}{*}{ Consumo habitual de frutas y verduras } & Sí & 153 & 34 \\
\hline & No & 296 & 66 \\
\hline \multirow[t]{2}{*}{ Antecedente de HTA } & Sí & 17 & 4 \\
\hline & No & 432 & 96 \\
\hline \multirow[t]{3}{*}{ Clasificación nutricional } & Bajo y normo peso $(<25)$ & 162 & 36 \\
\hline & $\begin{array}{l}\text { Sobrepeso } \\
\text { (IMC: 25-29.9) }\end{array}$ & 188 & 42 \\
\hline & Obesidad $(\geq 30)$ & 99 & 22 \\
\hline
\end{tabular}


TABLA 1. Características generales de la muestra incluida en el estudio ( $=449$ ) (continuación)

\begin{tabular}{|c|c|c|c|}
\hline \multicolumn{2}{|l|}{ Variable } & \multirow{2}{*}{$\begin{array}{c}\text { n } \\
194\end{array}$} & \multirow{2}{*}{$\begin{array}{l}\% \\
43\end{array}$} \\
\hline Obesidad abdominal & Sí & & \\
\hline & No & 255 & 57 \\
\hline \multirow[t]{2}{*}{ Antecedente de hiperglucemia } & Sí & 50 & 11 \\
\hline & No & 399 & 89 \\
\hline \multirow[t]{3}{*}{ Antecedente familiar de diabetes } & Padres o hermanos & 78 & 17 \\
\hline & Abuelos, tíos o primos & 299 & 67 \\
\hline & Ninguno & 72 & 16 \\
\hline \multirow[t]{2}{*}{ Tiempo diario en posición sedente } & $\geq 4$ horas /día & 175 & 39 \\
\hline & $<4$ horas/día & 274 & 61 \\
\hline \multirow[t]{2}{*}{ Consumo diario de bebidas azucaradas } & $\geq 5$ bebidas azucaradas/día & 22 & 5 \\
\hline & $<5$ bebidas azucaradas/día & 427 & 95 \\
\hline
\end{tabular}

HTA: hipertensión arterial; IMC: índice de masa corporal.

de 45 años, el 97\% tiene exceso de peso corporal ( $I M C \geq 25$ ), el $89 \%$ tiene obesidad abdominal, el $46 \%$ tiene antecedente de padres con diabetes y el $42 \%$ refiere haber tenido un examen de glucemia con resultados alterados en algún momento de su vida. Los resultados del cuestionario FINDRISC se muestran en la tabla 2. Los trabajadores con alto riesgo para diabetes reportaron un consumo promedio de bebidas azucaradas de 3.25 ( \pm 2.5 ) veces por día y un promedio de tiempo diario en posición sedente de $3.8( \pm 2.8)$ horas.

\section{Análisis bivariante}

Aspectos como el sexo femenino (OR: 0.947; IC 95\%: 0.562-1.595), la residencia en área urbana (OR: 0.931; IC 95\%: 0.507-1.708) y el cargo laboral administrativo (OR: 1.524; IC 95\%: 0.885-2.625) no mostraron asociación estadísticamente significativa con el riesgo para desarrollar diabetes. Por el contrario, el consumo de cinco o más bebidas azucaradas por día (OR: 5.455; IC 95\%: 2.274-13.081) y la permanencia durante cuatro o más horas por día en posición sedente (OR: 1.615; IC 95\%: 1.011-2.578) fueron variables con diferencias estadísticamente significativas que mostraron asociación con el riesgo para desarrollar diabetes. Los resultados se muestran en la tabla 3.

\section{Análisis multivariante}

Se construyó un modelo de regresión logística para documentar los factores asociados con el riesgo para desarrollar diabetes, que incluyó el tipo de cargo laboral (administrativo/operativo), el tiempo diario en posición sedente $(<4 / \geq 4 \mathrm{~h})$ y el consumo diario de bebidas azucaradas $(<5 / \geq 5$ ). No se confirmó una asociación estadísticamente significativa con el desempeño en un cargo administrativo (OR: 1.524; IC 95\%: 0.885-2.625; $\mathrm{p}=0.127$ ) ni con la permanencia en posición sedente durante cuatro o más horas por día (OR: 1.454; IC 95\%: 0.871-2.427; p = 0.153). Pero si se confirmó asociación estadísticamente significativa del riesgo para desarrollar diabetes con el consumo diario de cinco o más bebidas azucaradas (OR: 5.071; IC 95\%: 2.094-12.279; $\mathrm{p}=0.000$ ). 


\section{Rev ALAD. 2021;11}

TABLA 2. Resultados en el cuestionario FINDRISC (FINnish Diabetes RIsk SCore)

\begin{tabular}{|c|c|c|c|c|c|}
\hline \multicolumn{2}{|l|}{ Variable } & \multicolumn{2}{|c|}{ Alto riesgo para diabetes $(n=89)$} & \multicolumn{2}{|c|}{ Bajo riesgo para diabetes $(n=360)$} \\
\hline & & $n$ & $\%$ & $\mathrm{n}$ & $\%$ \\
\hline \multirow[t]{2}{*}{ Edad } & $\geq 45$ años & 47 & 53 & 66 & 18 \\
\hline & $<45$ años & 42 & 47 & 294 & 82 \\
\hline \multirow[t]{2}{*}{ Práctica habitual de actividad física } & Sí & 11 & 12 & 52 & 14 \\
\hline & No & 78 & 88 & 308 & 86 \\
\hline \multirow[t]{2}{*}{ Consumo habitual de frutas y verduras } & Sí & 33 & 37 & 120 & 33 \\
\hline & No & 56 & 63 & 240 & 67 \\
\hline \multirow[t]{2}{*}{ Antecedente de HTA } & Sí & 10 & 11 & 7 & 2 \\
\hline & No & 79 & 89 & 353 & 98 \\
\hline \multirow[t]{2}{*}{ Exceso de peso corporal } & $I M C \geq 25$ & 86 & 97 & 202 & 56 \\
\hline & $I M C<25$ & 3 & 3 & 158 & 44 \\
\hline \multirow[t]{2}{*}{ Obesidad abdominal } & Sí & 79 & 89 & 115 & 32 \\
\hline & No & 10 & 11 & 245 & 68 \\
\hline \multirow[t]{2}{*}{ Antecedente de hiperglucemia } & Sí & 37 & 42 & 13 & 4 \\
\hline & No & 52 & 58 & 347 & 96 \\
\hline \multirow[t]{2}{*}{ Antecedente de padres con diabetes } & Sí & 41 & 46 & 37 & 10 \\
\hline & No & 48 & 54 & 323 & 90 \\
\hline
\end{tabular}

HTA: hipertensión arterial; IMC: índice de masa corporal.

TABLA 3. Análisis bivariante

\begin{tabular}{|c|c|c|c|c|c|c|c|c|}
\hline \multicolumn{2}{|l|}{ Variable } & \multicolumn{2}{|c|}{$\begin{array}{l}\text { Alto riesgo para diabetes } \\
\qquad(\mathrm{n}=89)\end{array}$} & \multicolumn{2}{|c|}{$\begin{array}{l}\text { Bajo riesgo para diabetes } \\
\qquad(n=360)\end{array}$} & \multirow{4}{*}{$\begin{array}{l}\text { OR* } \\
0.947\end{array}$} & \multirow{4}{*}{$\begin{array}{c}\text { IC } 95 \% \\
0.56-1.59\end{array}$} & \multirow{4}{*}{$\begin{array}{c}\begin{array}{c}\text { Valor } p \\
\left(\text { prueba } \chi^{2}\right)\end{array} \\
0.837\end{array}$} \\
\hline & & \multirow{2}{*}{$\frac{n}{24}$} & \multirow{2}{*}{$\begin{array}{l}\% \\
27\end{array}$} & \multirow{2}{*}{$\frac{\mathbf{n}}{101}$} & \multirow{2}{*}{$\frac{\%}{28}$} & & & \\
\hline Sexo & Mujeres & & & & & & & \\
\hline & Hombres & 65 & 73 & 259 & 72 & & & \\
\hline \multirow[t]{2}{*}{ Lugar de residencia } & Urbano & 73 & 82 & 299 & 83 & \multirow[t]{2}{*}{0.931} & \multirow[t]{2}{*}{$0.50-1.70$} & \multirow[t]{2}{*}{0.817} \\
\hline & Rural & 16 & 18 & 61 & 17 & & & \\
\hline \multirow{2}{*}{$\begin{array}{l}\text { Tipo de cargo } \\
\text { laboral }\end{array}$} & Administrativo & 23 & 26 & 67 & 19 & \multirow[t]{2}{*}{1.524} & \multirow[t]{2}{*}{$0.88-2.62$} & \multirow[t]{2}{*}{0.127} \\
\hline & Operativo & 66 & 74 & 293 & 81 & & & \\
\hline \multirow{2}{*}{$\begin{array}{l}\text { Consumo diario } \\
\text { de bebidas } \\
\text { azucaradas }\end{array}$} & $\begin{array}{l}\geq 5 \text { bebidas } \\
\text { azucaradas/día }\end{array}$ & 12 & 13 & 10 & 3 & \multirow[t]{2}{*}{5.455} & \multirow[t]{2}{*}{$2.2-13.08$} & \multirow[t]{2}{*}{0.000} \\
\hline & $\begin{array}{l}<5 \text { bebidas } \\
\text { azucaradas/día }\end{array}$ & 77 & 87 & 350 & 97 & & & \\
\hline \multirow{2}{*}{$\begin{array}{l}\text { Tiempo diario en } \\
\text { posición sedente }\end{array}$} & $\geq 4$ horas / día & 43 & 48 & 132 & 37 & \multirow[t]{2}{*}{1.615} & \multirow[t]{2}{*}{$1.01-2.57$} & \multirow[t]{2}{*}{0.045} \\
\hline & < 4 horas/día & 46 & 52 & 228 & 63 & & & \\
\hline
\end{tabular}




\section{DISCUSIÓN}

La diabetes es prevenible si se detectan y controlan los factores de riesgo para desarrollarla ${ }^{19}$. Las actividades de tamización y prevención en el lugar de trabajo permiten llegar a una gran proporción de trabajadores adultos que habitualmente no asisten a los servicios médicos preventivos ${ }^{6}$. Una de las fortalezas de este estudio fue utilizar la herramienta FINDRISC en el contexto de los exámenes médicos de salud ocupacional para identificar el riesgo para diabetes de forma fácil, oportuna y a muy bajo costo en una población de trabajadores. De este modo, identificar los factores de riesgo para diabetes en población laboralmente activa permitirá diseñar programas de prevención en el contexto empresarial. Los programas de intervención de los factores de riesgo en el lugar de trabajo incentivarán a los trabajadores para que adopten estilos de vida más saludables, logren perder peso y reduzcan su nivel de riesgo para desarrollar diabetes ${ }^{20}$. La proporción de trabajadores identificados con alto riesgo para diabetes en este estudio (19.8\%) fue ligeramente superior a la encontrada por Godelieve ${ }^{21}$ en los trabajadores examinados por los servicios de salud ocupacional de Bélgica (17.5\%). También fue superior a la reportada por Rodríguez y Mendoza ${ }^{22}$ en Barranquilla (16.7\%), por Giraldo, et al. ${ }^{16}$ en Manizales (14.4\%) y por Montes-Ochoa, et al. ${ }^{23}$ en Medellín (9.8\%). La diferencia de proporciones con el estudio de Bélgica es mínima, se encuentra dentro del intervalo de confianza de las dos estimaciones y podría relacionarse con que en dicho estudio se utilizó la versión original finlandesa del cuestionario FINDRISC. La diferencia de proporciones con los estudios colombianos se relaciona con los diferentes puntos de corte en el cuestionario FINDRISC utilizados para clasificar el riesgo de diabetes. En el estudio de Manizales y en el estudio de Barranquilla utilizaron el valor 13 y en el estudio de Medellín el valor 14 como puntos de corte para clasificar el riesgo de DM2. La proporción de trabajadores con exceso de peso corporal en este estudio (64\%) es superior a la reportada para Colombia $(56.5 \%)^{24}$, pero es similar a la reportada por Gómez-Arbeláez ${ }^{19}$ en su estudio de validación del cuestionario FINDRISC en Colombia (67.5\%).

Múltiples investigaciones han demostrado que la ingesta de bebidas azucaradas incrementa la adiposidad, el peso corporal y el riesgo para desarrollar diabetes ${ }^{25}$. En este estudio encontramos que el consumo diario de bebidas azucaradas entre los sujetos con alto riesgo ( $3.25 \pm 2.5$ veces por día) fue significativamente superior a la de los sujetos con bajo riesgo ( $2.43 \pm 1.5$ veces por día). Se confirmó una asociación estadísticamente significativa entre el consumo diario de cinco o más bebidas azucaradas y el riesgo para desarrollar diabetes (OR: 5.0; $\mathrm{p}=$ 0.000 ); resultados similares, en donde el riesgo para desarrollar diabetes se incrementa proporcionalmente al consumo de bebidas azucaradas, han sido reportados por Aronow, et al. ${ }^{26}$ en Argentina y por Dussaillant, et al. ${ }^{27}$ en Chile. El tiempo diario en posición sedente se encuentra influenciado por factores individuales, laborales y organizacionales ${ }^{28}$. A pesar de la gran variabilidad interindividual en el tiempo de permanencia en posición sentado, múltiples estudios han podido demostrar la asociación directamente proporcional entre el tiempo sedente, el incremento en el IMC y el aumento en el riesgo para desarrollar diabetes ${ }^{29}$. En este estudio encontramos que el tiempo diario en posición sedente de los sujetos con alto riesgo ( $3.8 \pm 2.8$ horas por día) es ligeramente superior al tiempo diario en posición sedente de los individuos con bajo riesgo (3.2 \pm 2.7 horas por día). Sin embargo, la asociación entre el tiempo sedente y el riesgo para desarrollar diabetes no fue estadísticamente significativa (OR: 1.4; $\mathrm{p}=$ 0.153). Esta débil asociación entre el tiempo sedente y el riesgo para diabetes puede estar relacionada 
con factores individuales, sociales y laborales de los sujetos examinados o con un tamaño de muestra insuficiente.

Se tomaron todas las precauciones para controlar sesgos potenciales y garantizar la validez interna de esta investigación, sin embargo algunos aspectos podrían afectar la generalización de sus resultados. El tamaño de la muestra es pequeño y limitado a un único centro médico de salud ocupacional en la ciudad. Podrían obtenerse resultados más representativos y generalizables con una muestra de mayor tamaño y origen multicéntrico. En la muestra analizada la proporción de trabajadores del sexo masculino fue mayoritaria, sin embargo habría sido beneficioso obtener una muestra con proporciones similares entre hombres y mujeres. Para esta investigación se incluyeron trabajadores remitidos por empresas radicadas en Villavicencio, pero no se tomó en consideración la región del país de donde proviene cada trabajador, ni el grupo racial o étnico al cual pertenece. La frecuencia de consumo de bebidas azucaradas y el tiempo en posición sedente se estableció a partir de lo que recordó y declaró el trabajador, por tanto es posible la falta de exactitud en la frecuencia de consumo y en el tiempo sedente declarado por cada individuo.

En esta investigación se encontró una proporción de adultos con alto riesgo para diabetes que fue superior a la encontrada en otros estudios colombianos. Queda abierta una línea de investigación para que futuros estudios puedan confirmar este hallazgo y explorar otros factores de riesgo en población trabajadora. Futuros estudios deberán contar con muestras más grandes, de origen multicéntrico, con distribución por sexo equilibrada y tomar en consideración el origen étnico/racial de los individuos.

\section{CONCLUSIONES Y RECOMENDACIONES}

El objetivo principal de esta investigación fue identificar el riesgo para desarrollar diabetes y sus factores asociados en una población de trabajadores. Se evaluó una muestra representativa de la población laboral atendida en un centro médico de salud ocupacional en Villavicencio, allí se identificó una proporción elevada de trabajadores con alto riesgo para diabetes. Adicionalmente, se identificó la asociación entre este riesgo y el consumo de bebidas azucaradas. En la muestra examinada, fueron pocos los individuos que practican actividad física de forma habitual, el consumo de frutas y verduras fue escaso y la proporción de individuos con exceso de peso corporal fue superior a la estimación nacional. Como propuesta para el control de estos factores, se recomienda implementar actividades que promuevan la práctica de actividad física, la alimentación saludable y la reducción del peso corporal. Se confirmó una asociación significativa entre el consumo de bebidas azucaradas y el riesgo para desarrollar diabetes, por tanto se recomienda desestimular el consumo de bebidas azucaradas en los entornos laboral y extralaboral como medida efectiva para reducir el riesgo para diabetes. Aunque se detectó que el tiempo de permanencia en posición sedente de los individuos con alto riesgo es ligeramente superior que el tiempo sedente de los individuos con bajo riesgo, no fue posible confirmar una asociación estadísticamente significativa entre el tiempo diario en posición sedente y el riesgo para diabetes. Se recomienda realizar nuevas investigaciones, que incluyan mayores tamaños de muestra, para investigar con mayor exactitud dicha asociación. Tal como ha ocurrido en múltiples estudios a nivel internacional, se verificó la factibilidad, aplicabilidad y fácil interpretación del cuestionario FINDRISC para la identificación de individuos en riesgo para desarrollar 
diabetes. En esta oportunidad, el instrumento permitió conocer el nivel de riesgo en trabajadores durante el examen médico ocupacional. La tamización periódica del riesgo para diabetes en población trabajadora permitirá la implementación, en los centros de trabajo, de actividades que favorezcan el control de los factores de riesgo identificados.

\section{AGRADECIMIENTOS}

Los autores expresan su agradecimiento al Equipo Interdisciplinario para el mejoramiento de la Calidad de Vida en Salud Ocupacional (EQUIVIDA), a la Facultad de Ciencias de la Salud de la Universidad de los Llanos y a la Facultad de Medicina de la Universidad Cooperativa de Colombia, Villavicencio.

\section{FINANCIAMIENTO}

Los autores declaran que la investigación fue financiada con recursos propios.

\section{CONFLICTO DE INTERESES}

Los autores declaran no tener conflicto de intereses.

\section{RESPONSABILIDADES ÉTICAS}

Protección de personas y animales. Los autores declaran que para esta investigación no se han realizado experimentos en seres humanos ni en animales.

Confidencialidad de los datos. Los autores declaran que han seguido los protocolos de su centro de trabajo sobre la publicación de datos de pacientes.
Derecho a la privacidad y consentimiento informado. Los autores han obtenido el consentimiento informado de los pacientes y/o sujetos referidos en el artículo. Este documento obra en poder del autor de correspondencia.

\section{BIBLIOGRAFÍA}

1. International Diabetes Federation. Atlas de la Diabetes de la FID. 9. ${ }^{a}$ edición. Bruselas, Bélgica: International Diabetes Federation; 2019.

2. American Diabetes Association. Standards of medical care in diabetes - 2019. Diabetes Care. 2019;42 (Suppl 1):S71-S80.

3. Organización Mundial de la Salud. Informe mundial sobre la diabetes. Ginebra: Organización Mundial de la Salud; 2016.

4. Ampudia-Blasco F. Fallo celular beta y progresión de la diabetes mellitus tipo 2. Endocrinol y Nutr. 2003;50(7):274-9.

5. Aschner P. Epidemiología de la diabetes en Colombia. Av Diabetol. 2010;26:95-100.

6. Organizacion Mundial de la Salud. Prevención de las enfermedades no transmisibles en el lugar de trabajo a través del régimen alimentario y la actividad física. Ginebra: Organización Mundial de la Salud; 2008. pp.1-44.

7. López-Jaramillo P, Nieto-Martínez R, Aure-Fariñez G, Mendivil C, Lahsen $R$, Silva-Filho $R$, et al. Identification and management of prediabetes: results of the Latin America Strategic Prediabetes Meeting. Rev Panam Salud Pública. 2018;41:e172.

8. Lindström J, Tuomilehto J. The diabetes risk score: a practical tool to predict type 2 diabetes risk. Diabetes Care. 2003;26(3):725-31.

9. Ibrahim M, Tuomilehto J, Aschner P, Beseler L, Cahn A, Eckel RH, et al. Global status of diabetes prevention and prospects for action: A consensus statement. Diabetes Metab Res Rev. 2018;34(6):1-10.

10. England TN, Tuomilehto J, Lindström J, Eriksson JG, Valle TT, Hämäläinen $\mathrm{H}$, et al. Prevention of type 2 diabetes mellitus by changes in lifestyle among subjects with impaired glucose tolerance. N Engl J Med. 2001;344(18):1343-50.

11. López-Jaramillo P, Calderón C, Castillo J, Escobar ID, Melgarejo E, Parra GA. Prediabetes in Colombia: Expert consensus. Colomb Med. 2017;48(4):189-201.

12. Ministerio de Salud y Protección Social. Guía de práctica clínica para el diagnóstico, tratamiento y seguimiento de la diabetes mellitus en la poblacion mayor de 18 años. Bogotá: Ministerio de Salud y Protección Social, Instituto de Evaluación Tecnológica en Salud; 2016.

13. Campos N, Palomino G. FINDRISC, utility in the screening of diabetes, personalization and associations. Rev la Fac Med Humana. 2018;18(3): 64-74.

14. Congreso de Colombia. Ley 1562 de 2012. Diario Oficial de Colombia; 2012. pp. 1-22.

15. Ministerio de Protección Social. Resolución 2346 de 2007. Diario Oficial de Colombia; 2007.

16. Giraldo G, Moron S, Giraldo DF, Araque M, Duque G, Torres E. Riesgo de diabetes y prediabetes en Manizales, estudio RIDIMA. Rev Colomb Endocrinol Diabetes Metab. 2019;6(1):22-9.

17. Ministerio de Salud y Protección Social. Guía de práctica clínica para la prevención, diagnóstico y tratamiento del sobrepeso y la obesidad en adultos. Colombia: Ministerio de Salud y Protección Social; 2016.

18. Ministerio de Salud. Resolución 8430 de 1993. Diario Oficial de Colombia; 1993. pp. 1-19. 
19. Gómez D, Alvarado L, Ayala M, Forero L, Camacho P, López P. Evaluation of the Finnish Diabetes Risk Score to predict type 2 diabetes mellitus in a Colombian population: A longitudinal observational study. World J Diabetes. 2015;6(17):1337-44.

20. Barham K, West S, Trief P, Morrow C, Wade M, Weinstock RS. Diabetes prevention and control in the workplace. J Public Heal Manag Pract. 2011;17(3):233-41.

21. Godelieve J, Vandermissen M, Godderis L. Evaluation of the finnish diabetes risk score (FINDRISC) for diabetes screening in occupational health care. Int J Occup Med Environ Health. 2015;28(3):587-91.

22. Rodríguez $M$. Factores de riesgo de diabetes mellitus tipo 2 en poblacion adulta. Rev Colomb Endocrinol Diabetes y Metab. 2019;6(2):86-91.

23. Montes S, Serna K, Estrada S, Guerra F, Sánchez I. Caracterización de los factores de riesgo de diabetes mellitus tipo 2 mediante el test de FINDRISK en una población de 30 a 50 años de Medellín, Colombia. Med Lab. 2016;22(11):563-76.

24. Instituto Colombiano de Bienestar Familiar. Encuesta Nacional de la Situación Nutricional 2015. Colombia: Instituto Colombiano de Bienestar Familiar; 2015.
25. Gómez-Miranda LM. Revisión sistemática sobre el efecto del consumo de bebidas azucaradas sobre la adiposidad en adolescentes y adultos. Nutr Hosp. 2013;28(6):1792-6.

26. Aronow I, Lavanda I, Leal M. Hábitos de consumo de bebidas e infusiones sin alcohol y su relación en el estado nutricional en los empleados de mostrador de cuatro farmacias de la ciudad autónoma de Buenos Aires. Rev Chil Nutr. 2014;41(3):243-50.

27. Dussaillant C, Villarroel L, Marín PP. Una alimentación poco saludable se asocia a mayor prevalencia de síndrome metabólico en la población adulta chilena : estudio de corte transversal en la Encuesta Nacional de Salud 2009-2010. Nutr Hosp. 2015;32(5): 2098-104.

28. Mullane SL, Toledo MJL, Rydell SA, Feltes LH, Vuong B, Crespo NC, et al. Workplace sedentary behavior. Int J Behav Nutr Phys Act. 2017;14(1):1-10.

29. Peterman F, Garrido A, Díaz X. Tiempo destinado a estar sentado y niveles de adiposidad ¿Cuál es su efecto sobre el desarrollo de diabetes mellitus tipo 2? Rev Med Chile. 2018;(146):433-41. 